\title{
Attentional selection is biased towards controllable stimuli
}

\section{Greg Huffman $^{1}$ - James R. Brockmole ${ }^{1}$}

Published online: 12 March 2020

(C) The Psychonomic Society, Inc. 2020

\begin{abstract}
While the factors that contribute to individuals feeling a sense agency over a stimulus have been extensively studied, the cognitive effects of a sense of agency over a stimulus are little known. Here, we conducted three experiments examining whether attentional selection is biased towards controllable stimuli. In all three experiments, participants moved four circle stimuli, one of which was under their control. A search target then appeared on one of the stimuli. In Experiment 1, the target was always on the controlled stimulus, but we manipulated the degree of control the participant had. In Experiment 2, the controlled stimulus was the target on $50 \%$ of the trials. In Experiment 3, we used a central arrow cue to tell participants which arrow key to press (rather than using a free choice task) and made the controlled stimulus the target on $25 \%$ of the trials, making it nonpredictive of the target's location. Across the three experiments we found that visual selection was biased towards controllable stimuli. This attentional bias was larger when participants had full, rather than partial, control over the stimulus, indicating that sense of agency leads one to prioritize objects under their control. The fact that agency influenced attention when the controlled object contained the target in $100 \%, 50 \%$, and $25 \%$ of trials, and occurred even when participants needed to monitor the center of the display in order to know which arrow key to press, suggests that its influence does not depend on task relevance or volitional decision-making.
\end{abstract}

Keywords Selective $\cdot$ Perception and Action

When one's actions cause anticipated or predictable effects in the external world, we feel a sense of agency over those effects. The circumstances that lead a person to feel a sense of agency over his or her environment are well known (Haggard \& Chambon, 2012). For example, people are likely to feel a sense of agency over environmental changes that occur close in time and space to an action. What is less understood is the degree to which a sense of agency affects cognitive processing. Our goal with this report was to evaluate possible changes in attentional selection and control that emerge when observers obtain a sense of agency over particular aspects of the visual environment.

Attentional selection refers to the mechanism that selects portions of the environment for prioritized processing. Attentional selection is competitive in nature (Bichot, Rossi, \& Desimone, 2005; Bundesen, 1998; Desimone \& Duncan, 1995) and is affected by both physical stimulus salience as well as a person's current goals. For example, when looking for a friend in a crowd that you know is wearing a red shirt, attentional selection will be

Greg Huffman

gregory.h.huffman.ctr@mail.mil

1 University of Notre Dame, Notre Dame, IN, USA biased toward red things as well as things that obviously stand out from the environment. Goals can also drive attentional selection to areas where your friend is most likely to be. For example, you would probably start searching where you last saw your friend, or where people are most likely to be present, before searching less likely locations. Here, we examined whether attentional selection is also biased by a sense of agency.

In the work we report here, we connected a sense of agency to the degree of control one has over certain elements in an otherwise uncontrollable environment. Such situations are frequent in daily life. Consider your drive to work every morning. You have control over the speed and heading of your car, but not of the many cars you encounter on your way. How is attention allocated in such circumstances? On the one hand, we might predict that attention is biased away from the things we control given that the moment-to-moment spatiotemporal properties of such objects are both known and predictable, potentially reducing the cognitive demands needed to process them. On the other hand, attentional selection might be biased towards stimuli one has control over, as doing so would facilitate the learning of the associations between actions and their effects, which are vital to performing voluntary actions (Hauf, Elsner, \& Aschersleben, 2004). Additionally, exercising control over the environment is rewarding (Burger \& Cooper, 1979; Eitam \& Higgins, 2010, 2014; Higgins, 2012; Leotti, 
Iyengar, \& Ochsner, 2010; White, 1959), and reward value is known to influence attention (Anderson, Laurent, \& Yantis, 2011a, 2011b; Della Libera \& Chelazzi, 2006; Hickey, Chelazzi, \& Theeuwes, 2010, 2014; Miranda \& Palmer, 2014; Raymond \& O'Brien, 2009). Understanding the manner in which agency and control influence selective attention can therefore reveal more about the mechanisms that underlie attentional control.

Recently, Wen and Haggard (2018) reported the first and, to our knowledge only, study examining the relationship between agency and selective attention. They engaged participants in a dynamic visual search task, in which a target and several distractors moved through a display. People viewed displays containing four circular frames. When the participants moved the computer mouse, the circles moved. To manipulate a sense of agency, participants had varying levels of control over one of the circle's movements (i.e. when the participant moved the mouse left and then right, one of the stimuli would move left and then right with a probability between $0 \%$ and $100 \%$ ). The other three stimuli would follow the mouse movements $50 \%$ of time and move randomly the other $50 \%$ of the time. After a period of time, gaps appeared in all stimuli. Three of the stimuli had two gaps, and one had one gap. The task was to find the circle with one gap (which, in this experiment, was always the one linked to the mouse movements). In that experiment, participants found the targets faster as they had increasing levels of control, suggesting an attentional selection is, in fact, biased towards controllable stimuli. Two follow-up experiments further suggested that subsequent loss of control over a controlled stimulus has an additional prioritizing effect.

Wen and Haggard's (2018) study provides important evidence that attentional selection is biased towards a controllable stimulus when it is among noncontrollable stimuli, but their work also poses several new issues and questions, three of which we address in this report. First, for Wen and Haggard, the controllable stimulus was fully predictive of the target's eventual location. Thus, participants may have been incentivized to determine which object was under their control, as doing so would facilitate performance on the visual search task. In other words, because controllability was a cue to target identity, attentional biases and faster responses to the controllable object may have been a consequence of participants' strategies to identify the target via this route, rather than a general bias for controllability per se. Hence, we can ask, does the influence of agency on attention depend on its taskrelevance? Second, Wen and Haggard's participants were required to explicitly report the strength of their sense of agency over particular objects on each trial. Thus, their participants were aware of their (at least potential) control over some stimuli. Does the influence of agency depend on one's awareness of their control? Third, Wen and Haggard's participants chose when and how to change the motion of the stimuli in the display. Does the influence of agency require such volitional action selection, or can it result from stimulus-driven action selection (Herwig, Prinz, \& Waszak, 2007; Obhi \& Haggard, 2004; Pfister, Heinemann, Kiesel, Thomaschke, \& Janczyk, 2012)? Taken together, answering these three questions will reveal the extent to which the impact of agency on selective attention depends upon task relevance, strategy, and volitional action selection. In short, by moving beyond the circumstances previously investigated, we can to determine how robustly agency affects attentional selection.

We began our investigation by conceptually replicating Wen and Haggard's (2018) study and found evidence, as they did, to suggest that attentional selection is biased towards controlled stimuli. Our main contribution, accomplished in Experiments 2 and 3, was to examine the strength of this conclusion by seeking boundary conditions that may limit the attentional prioritization for processing controllable stimuli. Across these experiments, we focused on the degrees to which the effects of agency on selective attention depend upon task relevance, strategy, and volitional action selection. To preview our results, we found evidence of an attentional selection bias towards controllable stimuli regardless of all three of these factors, suggesting that agency exerts a strong and durable influence on attentional selection.

\section{Experiment 1}

The purpose of Experiment 1 was to replicate prior evidence suggesting that attentional selection is biased towards controllable stimuli. Our approach is based on the design used by Wen and Haggard (2018, Experiment 1). Each trial began with four stimuli on the screen. The participants then moved the stimuli using the arrow keys. Three of the stimuli moved randomly while, critically, the fourth stimulus's movement was probabilistically linked to the participants' key presses. On different trials, this stimulus moved according to the participant's key presses $0 \%, 40 \%, 60 \%$, or $100 \%$ of the time. After a given time period, one or two gaps appeared in each stimulus. The three randomly moving stimuli had two gaps, and the controlled stimulus had one gap. The task was to find the single gap stimulus. If attentional selection is biased towards controllable stimuli, then responses times should be inversely related to the degree of control the participant had over the target stimulus.

\section{Method}

\section{Participants}

Twenty-four University of Notre Dame undergraduate students participated in the experiment in exchange for course credit. All participants reported normal or correct-to-normal visual acuity and normal color vison. Previous research (Wen \& Haggard, 
2018), on agency and attention, used sample sizes of $\sim 20$, so we targeted a sample size of 24 to be conservative and account for the need to remove participants for poor performance.

\section{Stimuli and apparatus}

Participants completed the experiment using a PC connected to an LCD monitor (screen resolution: 1,920 $\times 1,080$ pixels, refresh rate: $60 \mathrm{~Hz}$ ). The experiment was programmed using MATLAB by MathWorks (The MathWorks, Natick, MA) with the Psychophysics toolbox (Kleiner, Brainard, Pelli, Ingling, Murray, \& Broussard, 2007). Stimuli consisted of four black circles $\left(\right.$ diameter $=\sim 2^{\circ}$ visual angle, pen width $=$ 3 pixels) that moved at a constant speed of 3.0 pix/s. Stimuli moved only horizontal or vertically (i.e., up, down, left, or right) within a uniform gray field (RGB: $128,128,128)$ that filled the screen, except for the bottom $1.4^{\circ}$, which was black. Various response prompts appeared within this lower black area, in white 30-point font. A standard QWERTY keyboard was used to collect responses from participants.

\section{Design and procedure}

The experimental procedure is illustrated in Fig. 1. Each trial began with the presentation of a black (RGB: 000, 000, 000) central fixation point $\left(\right.$ diameter $=\sim 0.3^{\circ}$ ). Participants were told to remain fixated on that point until the circles began to move. After 1,000 ms, the four black circles appeared at random locations within the display. Once the stimuli appeared, the participants pressed and held down their choice of one of the four arrow keys, which caused the four circles to move. Whenever a key was released, the motion of all circles would stop. Each time a participant pressed a new arrow key, each circle had the possibility of changing direction. ${ }^{1}$ Three of the four circles moved in the direction of the arrow key press $50 \%$ of the time (determined randomly for each circle). On the other $50 \%$ of key presses, the direction of travel for each of these three circles was chosen randomly at the time of the key press. The fourth target circle's movement direction was probabilistically determined by the participant's arrow key press. On a given trial, depending on condition, the target circle moved in the direction of the arrow key press $0 \%, 40 \%$, $60 \%$, or $100 \%$ of the time. When not moving in the direction of the key press, the target circle's direction was chosen randomly out of the remaining three possible directions of motion at the time of the key press.

After 2,000-3,500 ms of stimulus motion (specifically, a random selection amongst 16 possible durations separated by $100 \mathrm{~ms}$ intervals, i.e. $2,000,2,100,2,200, \ldots 3,500 \mathrm{~ms}), 0.3^{\circ}$

\footnotetext{
${ }^{1}$ When a circle reached an edge of the display, it "bounced" off of the edge and reversed direction. The circles, however, ignored and passed through each other.
}

gaps appeared in all four circle frames. The three circles that moved randomly had gaps on both the left and right sides. These circles constituted distractors in a visual search task. The target circle linked to the arrow key presses had only one gap that appeared on the left or right side. This constituted a search target that participants were to locate as quickly as possible. They pressed the space bar once they found the target. The gaps were only visible while the circles were moving; if the participants released an arrow key, the gaps disappeared until they again pressed a key.

Following the space-bar press, all stimuli stopped moving, the gaps disappeared, and a number (1-4, randomly assigned without replacement) appeared within each circle. The participants reported which circle was the target by pressing the corresponding number key. After reporting which circle was the target, participants responded to a controllability prompt: "Did you have more or less control over circle (the number they chose as the target) than the others? Less (1)| same (2) | more (3)," which they responded to using the 1,2 , and 3 keys on the number pad. The next trial began once this response was made.

Participants were not given explicit instructions as to how they should move the stimuli or how often they should change the motion of the circles. The instruction to report how much control they had over the search target at the end of the trial, however, incentivized exploration of the controllability of the stimuli. So, while it was possible to complete each trial by pressing and holding a single key, such behavior would handicap their ability to answer the controllability question. While we did not record participants' individual key presses in this experiment (we did record and analyze key presses in Experiment 2), as we will discuss below, response time patterns, coupled with accuracy on the control question, provide evidence that participants were indeed inclined to press multiple keys on each trial.

The experiment constituted a completely within-subjects design. Trials with different control probabilities $(0 \%, 40 \%$, $60 \%$, or $100 \%$ ) appeared equally often and in a random order. Control probability was crossed with the location of the target gap (left or right). Using a repetition factor of 15 , participants completed 120 trials.

\section{Results}

\section{Response times}

The mean response times are illustrated in Fig. 2a. We excluded trials in which errors were made in terms of target identification (5.6\% of trials), in which responses were made in fewer than $100 \mathrm{~ms}(0.3 \%)$, and/or response times were more than 2.5 standard deviations above each participant's mean response time $(2.2 \%)$. In sum, these trims excluded $7.9 \%$ of the data. We then analyzed the response-time data using a one-way 


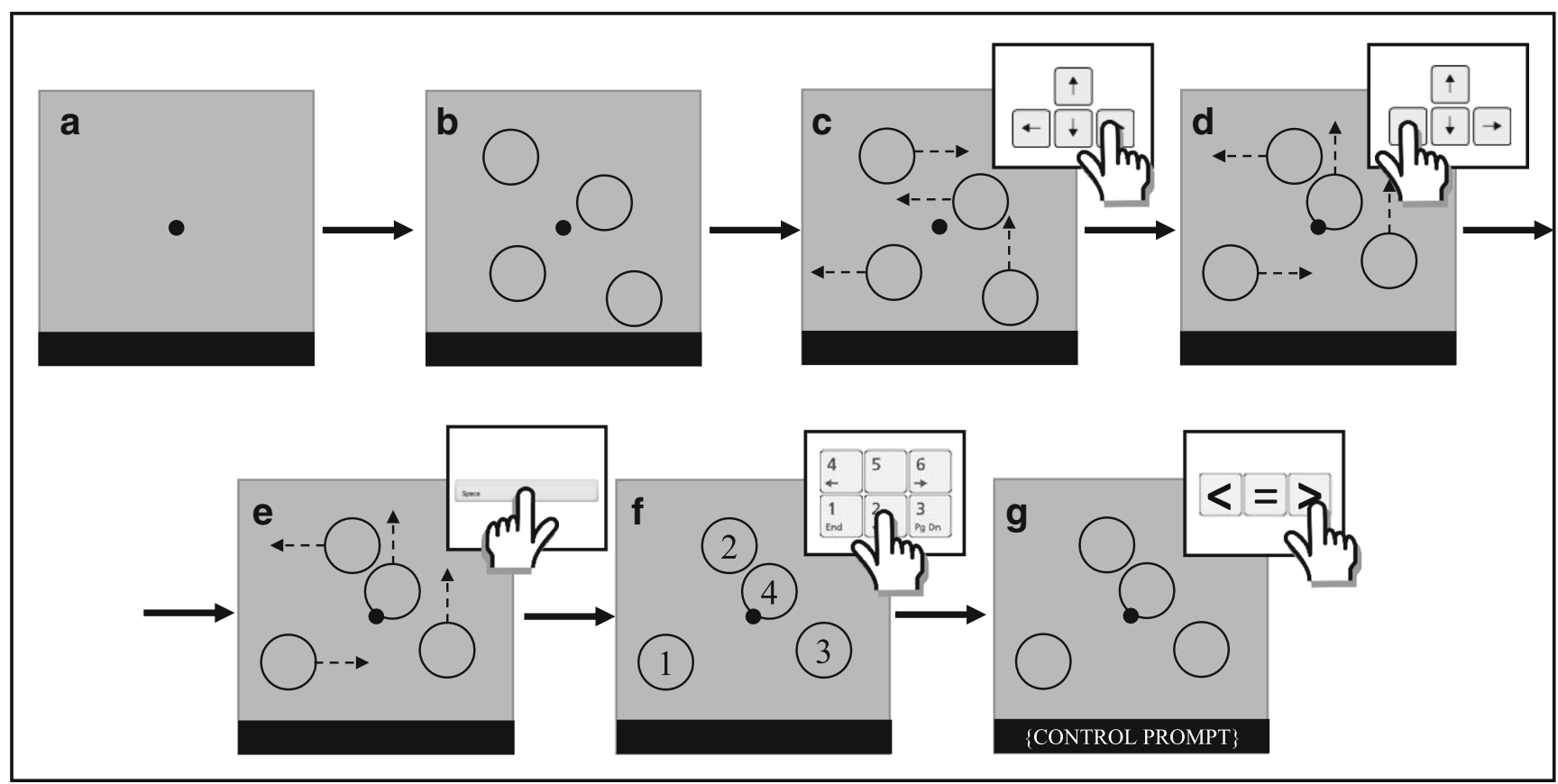

Fig. 1 Procedure used in Experiment 1. Fixation dot at center of screen (a) followed by appearance of four circles in random locations (b). Circles moved when participant pressed an arrow key (c) and changed direction when a different arrow was pressed (d). Search target is revealed and participant presses space bar when located (e). Circles stop moving and participant indicates which circle was target (f). Finally, participant participants respond to a control prompt (g)

controllability $(0 \%, 40 \%, 60 \%$, or $100 \%)$ as the factor and mean accuracy on the controllability prompt as the dependent measure. ${ }^{2}$ We observed an effect of controllability, $F(3,69)=8.082, p<.001, \eta_{p}^{2}=.260$. Accuracy in the $0 \%$ condition $(M \pm S E=70.7 \% \pm 3.6 \%)$ was higher than in the $40 \%$ condition $(58.9 \% \pm 3.2 \%), t(23)=2.144, p$ $=.043$, Cohen's $d_{z}=.438$. Accuracy in the $40 \%$ condition was no different than that in the $60 \%(63.8 \% \pm 2.1 \%)$ condition, $t(23)=1.104, p=.281$, Cohen's $d_{z}=.281$. Accuracy in the $100 \%(82.5 \% \pm 3.2 \%)$ condition was higher than that in the $60 \%$ condition, $t(23)=5.766, p<$ .001 , Cohen's $d_{z}=1.186$. These results suggest that participants were explicitly aware of how much control they had over the target compared with the distractors.

\section{Response time as a function of sense of agency}

Finally, we sought to determine whether participants' ability to report how much control they had over target

\footnotetext{
${ }^{2}$ This analysis was restricted to trials in which participants reported that they had either "less" or "more" control of the target stimulus; "same" was never the correct answer. Previous work (Wen \& Haggard, 2018) has used the "same" response as a measure of false alarms and calculated $d$ '. We elected not to do so because the design fails to meet the criteria for implementing signal-detection analyses in two ways. First, false alarms are defined as reporting the presence of a signal when there was none; hence, reporting "same" should be classified as a "miss" because the target stimulus was always more or less controllable than the distracting stimuli. Second, the experiment subjected to signaldetection analyses should include conditions that allow for hit, false-alarm, correct rejection, and miss responses, which is not the case in this design.
} 

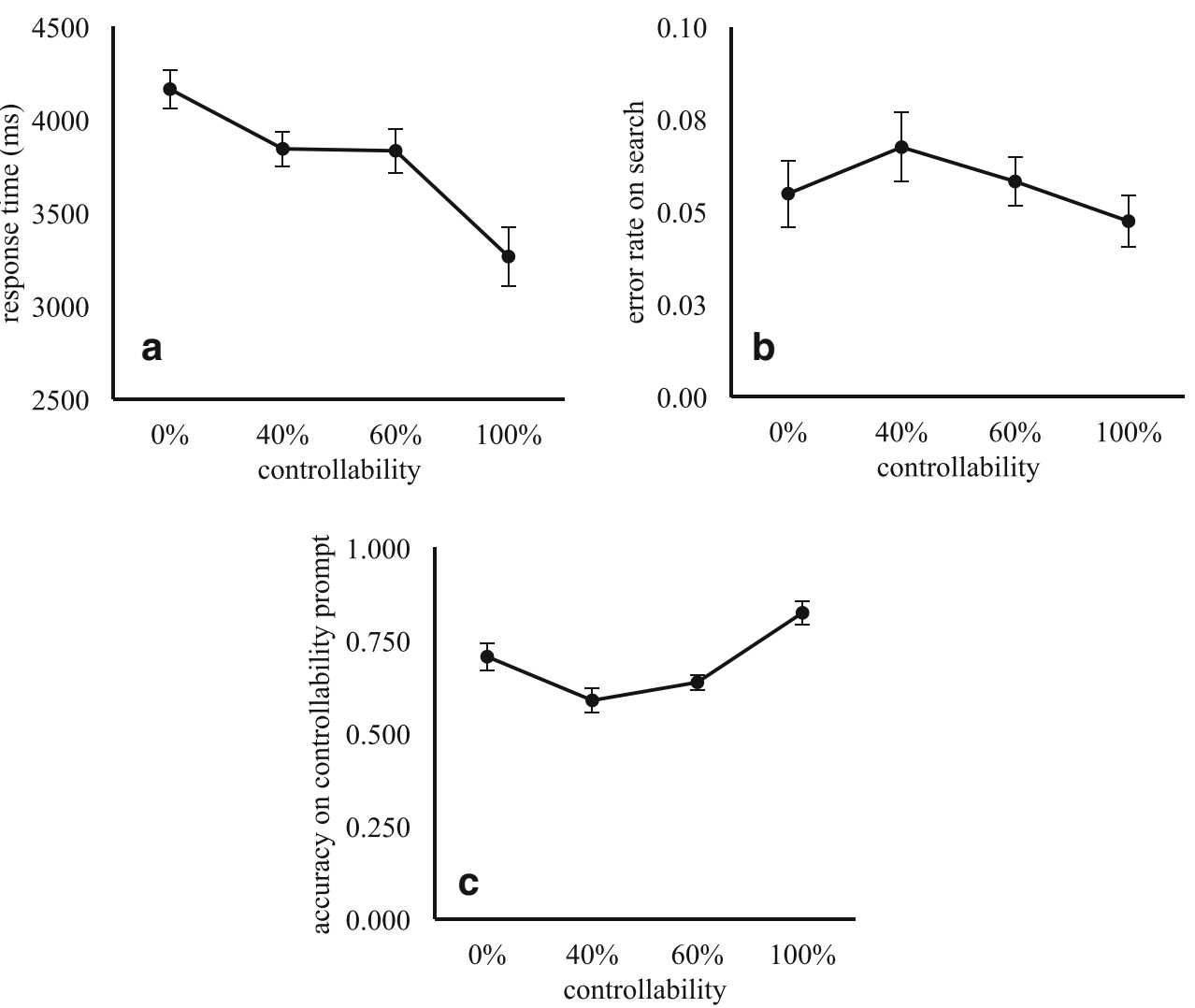

Fig. 2 Results from Experiment 1. a Mean response times on the search task as a function of controllability. b Error rate on the search task as a function of controllability. $\mathbf{c}$ Accuracy on the controllability prompt as a function of controllability. The error bars represent the within-subject standard error (Cousineau, 2005)

consistent with attentional selection being biased towards controllable stimuli. In the $0 \%$ control condition, the stimulus never moved in the direction of participants' key presses, and so attentional selection was biased towards the other three stimuli that did in fact move in the key press direction $50 \%$ of the time. In the $40 \%$ and $60 \%$ conditions, the target stimulus was about as likely as the noncontrollable stimuli to move in the direction of the key press. In these conditions, therefore, attention was selecting the controllable and noncontrollable stimuli at approximately equal rates. Finally, in the $100 \%$ condition, the target stimulus was constantly following the key press, creating a strong attentional selection bias towards it, leading to faster response times.

There are two notable limitations to this experiment, however. One is that the response times are exceptionally long relative to the difficulty of the task, suggesting that they may not be diagnostic of when participants found the search target. Instead, we suspect that participants continued to try to determine which object they were controlling (because they would be asked at the end of the trial) after they found the target. The second is, as in Wen and Haggard (2018), the controllable stimulus was always the search target, giving participants incentive to find the stimulus they are controlling and limiting the claims that can be 
made regarding an attentional bias. In Experiment 2, we revised the experimental design to address the long response times and ask whether this bias would survive in situations where agency is less task relevant. Then, in Experiment 3, we examined situations in which agency is not explicitly considered by participants nor the result of volitional action selection.

\section{Experiment 2}

In Experiment 1, the controllable stimulus was $100 \%$ predictive, incentivizing attentional selection of the controllable stimulus. In Experiment 2, the controllable stimulus was the target on $50 \%$ of the trials. If attentional selection is biased towards controllable stimuli, response times should be faster when the controlled stimulus is the target compared with when it is a distractor. Additionally, this difference should increase as a function of controllability $(0 \%, 40 \%, 60 \%, 100 \%)$. In addition to this change, we also implemented an additional experimental control. To address the long response times in Experiment 1, in Experiment 2 we had the stimuli freeze in place once the target gaps appeared. This limited participants' ability to determine their control over stimuli to the period of motion that occurred prior to the appearance of the search target.

\section{Method}

\section{Participants}

Twenty-four University of Notre Dame undergraduate students participated in the experiment in exchange for course credit. All participants reported normal or correct-to-normal visual acuity and normal color vison.

\section{Stimuli and apparatus}

The stimuli and apparatus were the same as in Experiment 1.

\section{Design and procedure}

The procedure for Experiment 2 was the same as in Experiment 1, with the following three exceptions. First, the controllable stimulus was the target on $50 \%$ of trials and a distractor on the other $50 \%$ of trials. Second, rather than waiting for a key press from participants, stimuli stopped moving once the gaps appeared. Finally, rather than asking the participants whether they had more or less control over the target stimulus than the other stimuli, we asked them to indicate which stimulus they had the most control over (to adapt to the stimulus linked to the key presses no longer always being the target). This adjustment was made in order for us to be able to determine whether participants were aware of which stimulus they were controlling, even though in half of the trials it was a distractor.

The experiment fully crossed each control probability $(0 \%, 40 \%, 60 \%$, or $100 \%)$, regardless of whether the target was the controllable stimulus or not, and in which side of the target the gap appeared (left or right). Using a repetition factor of 15, participants completed a total of 240 trials. Trials were presented in a different random order to each participant.

\section{Results}

\section{Response time}

The mean response times are illustrated in Fig. 3a. We excluded trials in which errors were made (6.9\% of trials), in which responses were made in fewer than $100 \mathrm{~ms}(0.4 \%)$, and/or in which response times were slower than 2.5 standard deviations from each participant's mean response time (1.7\%). In sum $9.0 \%$ of the data were excluded. We conducted a 4 (controllability: $0 \%, 40 \%, 60 \%$, and $100 \%) \times 2$ (target location: the controllable stimulus or a noncontrollable stimulus) repeated-measures ANOVA. We observed a reliable main effect of controllability, $F(3,69)=14.776, p<.001, \eta_{p}^{2}=.391$. Response times in the $0 \%$ controllability condition $(1,472 \pm$ $54 \mathrm{~ms}$ ) were slower than the $40 \%$ controllability condition $(1,407 \pm 48 \mathrm{~ms}), t(23)=2.425, p=.024$, Cohen's $d_{z}=.495$. Response times in the $40 \%$ controllability condition were statistically indistinguishable from those in the $60 \%$ controllability condition $(1,373 \pm 45 \mathrm{~ms}), t(23)=1.665, p=.109$, Cohen's $d_{z}=.340$. Response times in the $100 \%$ controllability condition $(1,290 \pm 45 \mathrm{~ms})$ were faster than the response times in the $60 \%$ controllability condition, $t(23)=3.164, p=.004$, Cohen's $d_{z}=.646$. We also observed a main effect of target type with faster response times when the controllable stimulus was the target $(1,321 \pm 44 \mathrm{~ms})$ compared with when the noncontrollable stimulus was the target $(1,450 \pm 49 \mathrm{~ms}), F(1,23)$ $=36.866, p<.001, \eta_{p}^{2}=.616$.

In addition to the main effects of each factor, controllability and target location reliably interacted, $F(3,69)=19.864, p<$ $.001, \eta_{p}^{2}=.463$. In the $0 \%$ control condition, response times were no different depending on whether the target was a controllable $(1,471 \pm 54 \mathrm{~ms})$ or noncontrollable stimulus $(1,474 \pm$ $60 \mathrm{~ms}), t(23)=.081, p=.936$, Cohen's $d_{z}=.017$. In the $40 \%$ control condition, again there was no difference between when the target was a controllable $(1,386 \pm 51 \mathrm{~ms})$ versus noncontrollable stimulus $(1,429 \pm 50 \mathrm{~ms}), t(23)=1.474, p=$ .154 , Cohen's $d_{z}=.301$. In the $60 \%$ control condition, response times were significantly faster when targets were controllable $(1,307 \pm 44 \mathrm{~ms})$ compared with when they were noncontrollable $(1,438 \pm 56 \mathrm{~ms}), t(23)=2.927, p=.008$, 

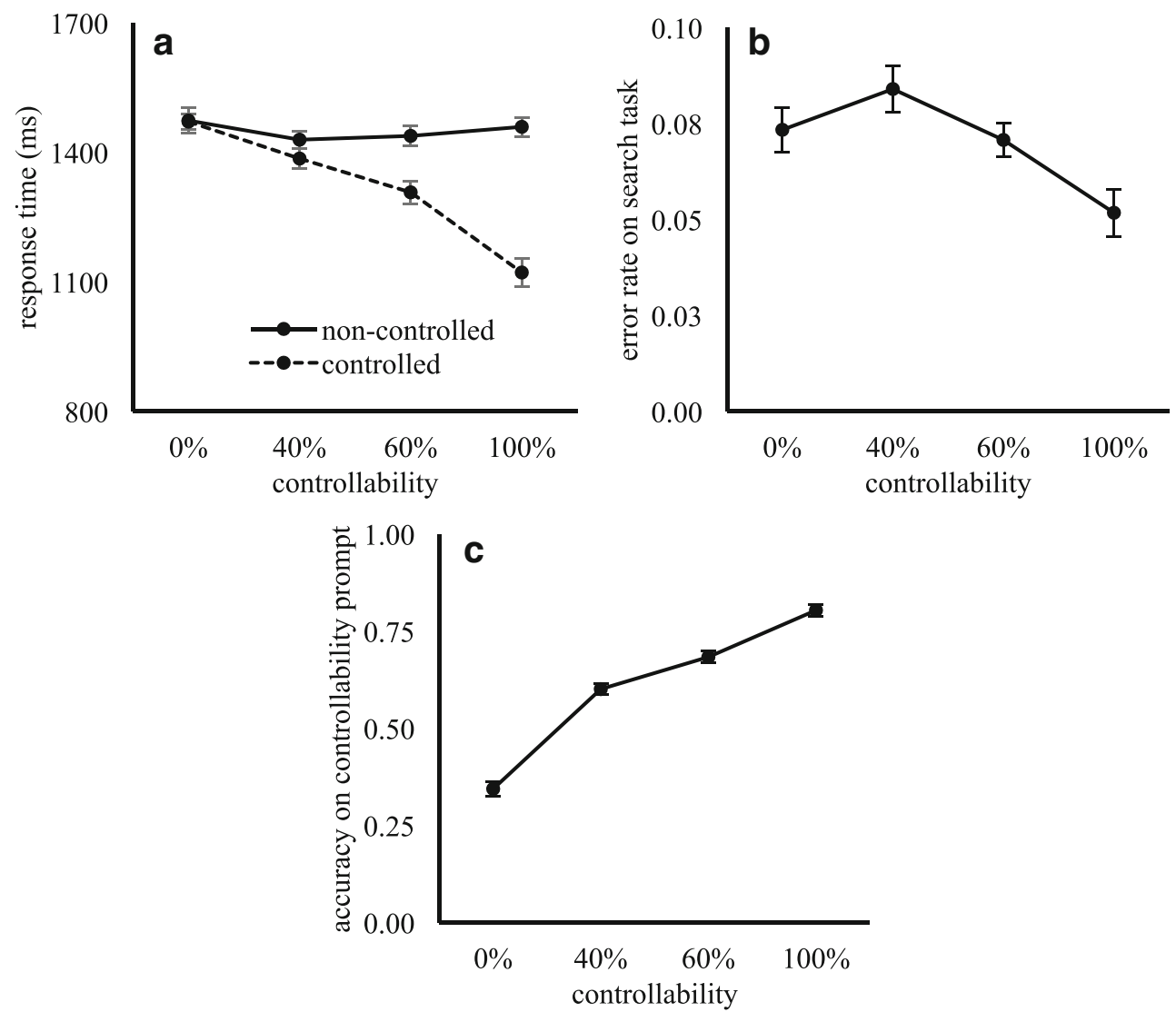

Fig. 3 Results from Experiment 2. a Mean response times by level of controllability and target location. The error bars represent the $95 \%$ confidence interval of the difference between the noncontrolled and controlled conditions at each controllability level (Pfister \& Janczyk,

Cohen's $d_{z}=.597$. Finally, in the $100 \%$ control condition, response times were significantly faster when targets were controllable $(1,122 \pm 50 \mathrm{~ms})$ compared with when they were noncontrollable $(1,459 \pm 47 \mathrm{~ms}), t(23)=9.301, p<.001$, Cohen's $d_{z}=1.898$.

\section{Errors}

The mean error rates on the search task are illustrated in Fig. 3b. Error rates were also analyzed using a 4 (controllability: $0 \%, 40 \%, 60 \%$, and $100 \%) \times 2$ (target location: the controllable stimulus or a noncontrollable stimulus) repeated-measures ANOVA. We observed a main effect of controllability, $F(3$, 69) $=4.316, p=.008, \eta_{p}^{2}=.158$. This was solely driven by higher error rates in the $60 \%$ control condition $(7.1 \% \pm 0.8 \%)$ compared with the $100 \%$ control condition $(5.2 \% \pm 0.7 \%)$, $t(23)=2.818, p=.010$, Cohen's $d_{z}=.575$. Error rates did not significantly differ between the $0 \%$ control condition $(7.3 \% \pm 1.0 \%)$ and the $40 \%$ control condition $(8.4 \% \pm$ $1.1 \%), t(23)=1.246, p=.225$, Cohen's $d_{z}=.254$, nor did they differ in the $40 \%$ control condition and the $60 \%$ control condition $(7.1 \% \pm 0.8 \%), t(23)=1.437, p=.164$, Cohen's $d_{z}=$
2013). b Error rate on the search task as a function of controllability. c Accuracy on the controllability prompt as a function of controllability. The error bars represent the within-subject standard error (Cousineau, 2005)

293. Neither the main effect of target type, $F(1,23)=2.148, p$ $=.156, \eta_{p}^{2}=.085$, nor the interaction of these factors, $F(3,69)$ $=.065, p=.978, \eta_{p}^{2}=.003$, were reliable. Importantly, these effects do not reflect a speed-accuracy trade-off when considered with the response-time data.

\section{Sense of agency}

The mean accuracies on the control prompt are illustrated in Fig. 3c. To determine whether participants were able to report how much control they had over the target, we conducted a one-way, within-subjects ANOVA, with controllability as the factor and mean accuracy on the controllability prompt as the dependent measure. We observed an effect of controllability, $F(1,23)=112.343, p<.001, \eta_{p}^{2}=.83$, indicating that participants were sensitive to the agency manipulation. Accuracy in the $0 \%$ control condition $(34.4 \% \pm 2.8 \%)$ were lower than those in the $40 \%$ condition $(60.0 \% \pm 2.2 \%)$. Accuracy in the $60 \%$ condition $(68.3 \% \pm 1.9 \%)$ was higher than accuracy in the $40 \%$ condition. Accuracy in the $100 \%$ condition $(80.4 \% \pm$ $1.4 \%$ ) was higher than that in the $60 \%$ condition. 


\section{Response time as a function of sense of agency}

We also considered whether the size of the response-time advantage for controllable targets was correlated with the participants' ability to report how much control they had over the target. To do so, we calculated the difference between the control and noncontrollable conditions for each level of control and fit a line to those difference scores. We compared the slope of that line to that of a line fit to the accuracy of control response data. Unlike in Experiment 1, we found no significant relationship between participants' abilities to report how much control they had over targets and the size of the controllable target response time advantage, $r=.177, p=.409$.

\section{Number of key presses and performance}

Finally, we examined the participants' key press behavior and its relationship to the controllability bias and the ability to determine how much control they had to move the target stimulus. On average, participants made 6.6 key presses per trial (range: $3.2-15.5$ ). There was a negative correlation between number of key presses per trial and the size of the controllability bias across the controllability conditions, $r(22)=-.566$, $p=.004$. While this would be a perplexing relationship, when viewing a scatter plot of the data, it was apparent that there was a high-leverage outlier (15.5 key presses per trial and a slope of -288). If that outlier is removed, the correlation becomes nonsignificant, $r(21)=-.211, p=.344$. We next looked at whether the number of the key presses per trial correlated with participants' accuracy at reporting how much control they had over a stimulus. Here, we found no significant relationship, $r(22)=-.178, p=.404$. The fact that the size of the controllability bias was unrelated to the number of key presses suggests that the attentional bias toward agency may not be graded in nature, but rather emerges in full once the controllable stimulus was determined and does not strengthen with more stimulus manipulation.

\section{Discussion}

As with Experiment 1, Experiment 2's results are consistent with attentional selection being biased towards controllable stimuli. When participants had $60 \%$ or $100 \%$ control over a stimulus, response times were faster when the controllable stimulus was a target compared with when it was a distractor. This difference was larger in the $100 \%$ control condition compared with the $60 \%$ control condition. Given that controllability was no longer $100 \%$ predictive of target location, this data strengthens the argument that attentional selection is biased towards controllable stimuli. In Experiment 3, we reduced the relationship between agency and target location to chance, did not ask participants to identify the controlled stimulus, and removed volitional action selection regarding the control of objects.

\section{Experiment 3}

Our goal for Experiment 3 was to provide a strong test of the hypothesis that attentional selection is biased towards controllable stimuli. To that end, we made a few changes to the method. First, the controllable stimulus was now nonpredictive of the target's location, with the target appearing on the controlled stimulus on $25 \%$ of trials. Second, whereas in previous experiments we allowed the participants to choose which arrow keys to press, here, we had them monitor a centrally presented arrow cue that told them what response to make. Because this manipulation makes it so participants are no longer intentionally selecting which direction to move the controllable stimulus (and monitoring which stimulus moved in that direction), this should reduce the relevance of the controllable stimulus such that they are less likely to actively seek out which stimulus they are controlling. Third, in Experiment 3 we no longer informed the participants that they were in control of one of the stimuli and did not probe them about control at any time. Finally, for the sake of simplicity, we dropped the controllability manipulation such that on all trials they had $100 \%$ control over one stimulus.

\section{Method}

\section{Participants}

Twenty-four University of Notre Dame undergraduate students participated in the experiment in exchange for course credit. All participants reported normal or correct-to-normal visual acuity and normal color vison.

\section{Stimuli and apparatus}

The stimuli and apparatus were the same as in Experiments 1 and 2 .

\section{Design and procedure}

The procedure of Experiment 3 replicated Experiment 2, with the following three exceptions. First, the target was the controllable stimulus on $25 \%$ (rather than $50 \%$ ) of the trials to make control nonpredictive of target location. Second, we gave the participants instruction as to which arrow key to press using an arrow cue in the center of the display (a "<,", ">," " ," or a " "). The arrow direction was chosen randomly and remained the same direction for $500-1,000 \mathrm{~ms}$ before changing directions. Each time the arrow direction changed, the stimuli would stop moving until the participant pressed the 
new cued arrow key. On average, given the length of trials $(M$ $=2,750 \mathrm{~ms})$ and how often the key presses changed $(M=750$ $\mathrm{ms}$ ), participants made 3.6 trials per trial. This is somewhat less than in Experiment 2, but in pilot testing we found that changing the arrow direction more frequently made the task very difficult for participants to manage. Finally, we no longer informed the participants that they had control over one of the stimuli.

The experiment constituted a completely within-subjects design. Whether the target was controllable ( $25 \%$ controllable, $75 \%$ noncontrollable) was crossed with the location of the target gap (left or right). Using a repetition factor of 20, participants completed 160 trials.

\section{Results}

\section{Response times}

The mean response times are illustrated in Fig. 4, left panel. We discarded trials in which errors were made ( $4.2 \%$ of trials), in which responses were made less than $100 \mathrm{~ms}(0.0 \%)$, and/ or were slower than 2.5 standard deviations from each participant's mean response time (1.3\%). In sum $5.5 \%$ of trials were excluded via these trims. We conducted a paired-samples $t$ test comparing response times when targets were controllable versus noncontrollable. We found that response times were faster when targets were controllable $(1,252 \pm 38 \mathrm{~ms})$ than when they were noncontrollable $(1,299 \pm 33 \mathrm{~ms}), t(23)=2.494, p$ $=.020$, Cohen's $d_{z}=.509$.

\section{Errors}

The mean error rates in the search task are illustrated in Fig. 4, right panel. A paired-samples $t$ test, with error rates as the dependent variable, indicated no difference in the error rates when targets were controllable $(3.6 \% \pm 0.4 \%)$ compared with

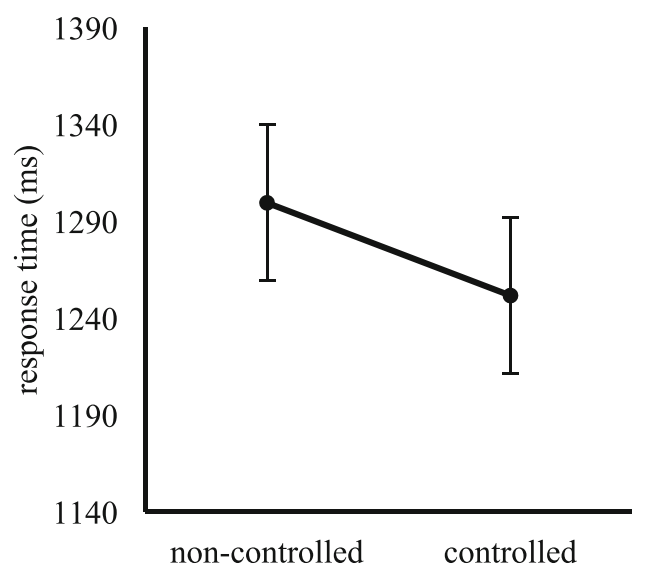

Fig. 4 Results from Experiment 3. Left panel: Mean response times by target location for Experiment 3. Right panel: Error rate on the search task. The error bars represent the $95 \%$ confidence interval of the when they were noncontrollable $(4.3 \% \pm 0.7 \%), t(23)=1.037$, $p=.310$, Cohen's $d_{z}=.212$.

\section{Discussion}

In Experiment 3, we continued to observe faster response times when targets appeared on controllable stimuli, even though the controllable stimulus was nonpredictive of target location and participants were not intentionally moving the stimuli in order to determine which stimulus they were controlling. This is strong evidence that attentional selection is biased towards controllable stimuli. Even when participants were not told that they were controlling a stimulus and had no incentive to look at the controllable stimulus compared with any of the other stimuli, they responded faster when it was the target, indicating an attentional bias towards controllable stimuli.

\section{General discussion}

Previous research has suggested that attentional selection is biased towards controllable stimuli (Wen \& Haggard, 2018). Here, we looked to build upon those findings in order to establish to what extent this bias depends upon that control being task relevant, brought to awareness, and the result of volitional decision-making. To begin, we conceptually replicated Experiment 1 from Wen and Haggard (2018) in which participants had varying levels of control $(0 \%, 40 \%, 60 \%$, or $100 \%$ ) over one stimulus, while the others moved in the direction of the key press $50 \%$ of time and in a random direction the other $50 \%$ of the time. The controllable stimulus was always the target, and the participants needed to report how much control they had over the target compared with the other stimuli. As did Wen and Haggard, under these conditions, we observed faster response times as a function of increasing

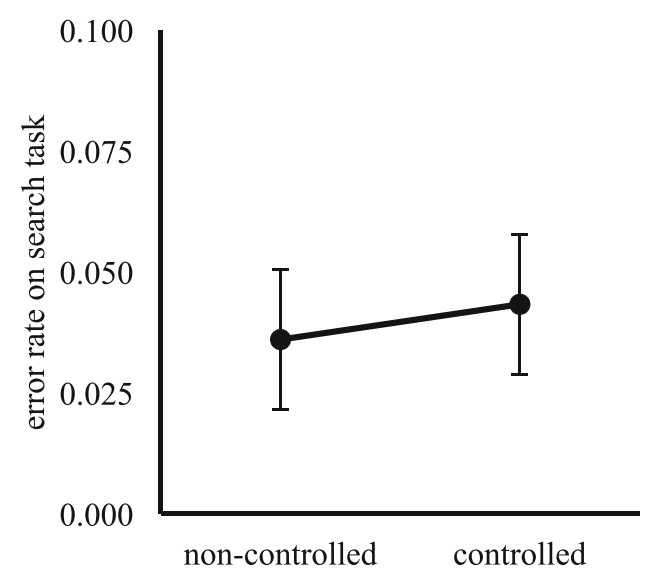

difference between the non-controlled and controlled conditions at each controllability level (Pfister \& Janczyk, 2013) 
control over the stimulus, suggesting a selection bias towards that stimulus. In Experiment 2, we modified our methods so that the controllable stimulus was the target on only $50 \%$ of the trials and again replicated the selection bias towards the controllable stimulus. In our critical Experiment 3, we reduced the probability that the controllable stimulus would be the target from $50 \%$ to $25 \%$, making it nonpredictive of the search target's location, told the participants which arrow key responses to make, and no longer told them they were controlling one of the stimuli, and continued to find an attentional selection bias towards controllable stimuli. Thus, humans are sensitive to the changes their actions cause in the environment, and selective attention is biased towards those changes.

Interestingly, across our three experiments, the magnitudes of the observed biases toward controllable stimuli were not equivalent. As the relationship between target identity and stimulus control decreased (100\% in Experiment 1, 50\% in Experiment 2, and 25\% in Experiment 3), so too did the size of participants' bias. We think it is likely that responses in Experiments 1 and 2 were a product of both an attentional bias towards controllable stimuli and strategies that took advantage of that controllability to predict eventual target locations. As we reduced participants' ability to deploy those strategies, the processing benefits enjoyed by controlled stimuli were similarly diminished. That there was still evidence of an attentional bias in Experiment 3 when finding the controllable stimulus offered no benefit in the search task provides the strongest evidence to date that there an independent attentional bias towards controllable stimuli does exist outside of strategic control.

For attentional selection to be biased towards controllable stimuli, there must first be a mechanism sensitive to environmental changes caused by our actions (action outcomes). Wen and Haggard (2018) suggested we accomplish this actionoutcome detection by comparing our expected action outcomes with the actual outcomes (see also, Blakemore, Frith, \& Wolpert, 1999; Blakemore, Wolpert, \& Frith, 1998, 2002; Frith, Blakemore, \& Wolpert, 2000; Wolpert \& Flanagan, 2001; Wolpert \& Ghahramani, 2000). In our study, for example, the expected action outcome (based on a priori experience) of pressing the left arrow key would be some sort leftward movement. Having pressed the left arrow key, a comparator unit then determines which outcomes are likely to have resulted from our action and which are not. Our findings are consistent with Wen and Haggard's suggestion while also expanding upon their findings. Wen and Haggard found people to be sensitive to their action outcomes when those actions were task relevant. We have found that this continues to be the case, even when the actions are nonintentional (cued by an arrow rather than free choice) and the action outcomes are task irrelevant. From a developmental perspective, it makes sense that a mechanism for detecting action outcomes would not be retrained to detecting only expected outcomes. This is because linking outcomes to our actions is critical for the development of motor coordination (Gaudiano \& Grossberg, 1991; Rolf, Steil, Gienger, 2011), our sense of space (Philipona, O'Regan, \& Nadal, 2003), and color (Bompas \& O'Regan, 2006a, 2006b), fine shape (Herwig, Weiß, \& Schneider, 2015), and high spatial frequency (Herwig \& Schneider, 2014) perception in the periphery where, physiologically, rods are mostly insensitive to such information. Indeed, at the broadest perspective, some argue that linking actions and outcomes underlies the development of visual consciousness (Clark, 2013; O'Regan $\&$ Noë, 2001). Given this importance of linking actions and outcomes, the need for a mechanism highly sensitive to action outcomes is apparent and is likely facilitated by biased attentional selection of such action outcomes.

With strong evidence in hand that attention is biased toward aspects of the visual environment within our control, several new issues come to light. First, while a bias towards our actions' outcomes could facilitate learning new actionoutcome associations by prioritizing those stimuli for further processing (Haggard \& Chambon, 2012), a bias away from action outcomes could facilitate exploring less predictable aspects of the environment that may contain unknown benefit (or threat). While our data are consistent with the first possibility, they do not rule out the second. It is possible that once agency is established, attentional selection processes may be freed to explore the environment. If so, selection biases towards controllable aspects of the environment that are evident early on while control is being established might reduce over time. That prediction would be consistent with a recent gaze control model (i.e., one that predicts overt attentional allocation), in which when and where the eyes move is based upon the expected value of keeping the eyes were they are versus the expected value of moving them to a new location (Tatler, Brockmole, \& Carpenter, 2017). Applied here, maintaining attention on the controllable stimulus loses informative value over time relative to investigating other locations (see also, the balance between exploitation of known information and searching for novel information; Hills et al., 2015). To test this possibility, one would need to use longer movement phase time intervals than the 2,000-3,500 ms we used here. Furthermore, these longer intervals could be used in tandem with eye tracking to monitor the deployment of overt attention as control is gained/lost over a stimulus.

A second intriguing line of future research would determine the manner in which the attentional bias associated with agency is computed. Throughout this report, we have assumed that agency over an object is established by accumulating information over multiple interactions with the visual world. In fact, we manipulated participants' cumulative experience by varying the global probabilities that governed the extent to which participants had control of a stimulus throughout a trial. This led us to argue that once an observer has a consistent sense of agency over an object, that object is prioritized for 
processing. Admittedly, there is an alternative possibility that is also consistent with our results - namely, that the attentional bias associated with agency is a more transient phenomenon. Within our experiential paradigm, with each individual key press, multiple stimuli moved according to the participant's action input (the controlled stimulus simply did so with more consistency over multiple key presses). It is possible that the agency bias arose from an "amnesiac" process by which attention is allocated to the stimulus (or subset of stimuli) that most recently moved in the direction of the latest key press. Differentiating these possibilities is beyond our ability with our existing data, but it would elucidate the computational mechanism and time scale over which one's actions and their outcomes influence attentional control.

Third, future research on agency and attentional control should determine the ways in which the bias described here interacts with other well-known biases of attentional control. We have shown how agency and search strategies can be additive. How are the effects of agency influenced by other exogenous and endogenous factors that control attention? Is agency a kind of involuntary capture effect that survives other forms of cuing? Or is the bias more nuanced, competing with or augmenting other factors? Understanding these interactions will reveal the relative importance that agency plays within the constellation of factors known to bias attention.

Fourth, and finally, it is unclear to what extent agency is a factor that biases attention per se, or if it is a specific example of a broader attentional mechanism. Having control over an object gives one the ability to predict its future behavior, motion, or location, for instance. Could this predictive aspect of agency be the root cause of the bias we observed in this study? Or does agency give an object a sort of "singleton" status where its motion "stands out" against other objects in the display (cf. Lavie \& De Fockert, 2005; Theeuwes, 1992, 1994). Other possibilities certainly exist, and it will be important for future work to determine if agency is indeed an independent mechanism via which attention is controlled.

Despite the many new questions we have posed, and others will surely propose in the future, we found evidence that attentional selection is biased towards controllable stimuli and that this bias occurs even when controllability is task irrelevant, is not reinforced by the experimenters, and is divorced from volitional decision-making. These findings highlight the strong link between actions, perception, and attention. When a strong relationship between actions and perceptual events occur, attentional selection is biased towards the perceptual events. This is possibly to highlight the link between specific actions and outcomes in order to facilitate the development of motor and perceptual competency.

Open practices statement The data and or materials used for the current study are available upon request, and none of the experiments was preregistered.

\section{References}

Anderson, B. A., Laurent, P. A., \& Yantis, S. (2011a). Learned value magnifies salience-based attentional capture. PLOS ONE, 6(11), e27926.

Anderson, B. A., Laurent, P. A., \& Yantis, S. (2011b). Value-driven attentional capture. Proceedings of the National Academy of Sciences of the United States of America, 108(25), 10367-10371. https://doi. org/10.1073/pnas.1104047108

Bichot, N. P., Rossi, A. F., \& Desimone, R. (2005). Parallel and serial neural mechanisms for visual search in macaque area V4. Science, 308(5721), 529-534.

Blakemore, S. J., Frith, C. D., \& Wolpert, D. M. (1999). Spatio-temporal prediction modulates the perception of self-produced stimuli. Journal of Cognitive Neuroscience, 11(5), 551-559.

Blakemore, S. J., Wolpert, D. M., \& Frith, C. D. (1998). Central cancellation of self-produced tickle sensation. Nature Neuroscience, 1(7), 635 .

Blakemore, S. J., Wolpert, D. M., \& Frith, C. D. (2002). Abnormalities in the awareness of action. Trends in Cognitive Sciences, 6(6), 237242.

Bompas, A., \& O'Regan, J. K. (2006a). Evidence for a role of action in colour perception. Perception, 35(1), 65-78.

Bompas, A., \& O'Regan, J. K. (2006b). More evidence for sensorimotor adaptation in color perception. Journal of Vision, 6(2), 5-5.

Bundesen, C. (1998). A computational theory of visual attention. Philosophical Transactions of the Royal Society of London, Series B: Biological Sciences, 353(1373), 1271-1281.

Burger, J. M., \& Cooper, H. M. (1979). The desirability of control. Motivation and Emotion, 3(4), 381-393.

Clark, A. (2013). Whatever next? Predictive brains, situated agents, and the future of cognitive science. Behavioral and Brain Sciences, 36(3), 181-204.

Cousineau, D. (2005). Confidence intervals in within-subject designs: A simpler solution to Loftus and Masson's method. Tutorials in Quantitative Methods for Psychology, 1(1), 42-45.

Della Libera, C., \& Chelazzi, L. (2006). Visual selective attention and the effects of monetary rewards. Psychological Science, 17(3), 222227.

Desimone, R., \& Duncan, J. (1995). Neural mechanisms of selective visual attention. Annual Review of Neuroscience, 18(1), 193-222.

Eitam, B., \& Higgins, E. T. (2010). Motivation in mental accessibility: Relevance of a representation (ROAR) as a new framework. Social and Personality Psychology Compass, 4(10), 951-967.

Eitam, B., \& Higgins, E. T. (2014). What's in a goal? The role of motivational relevance in cognition and action. Behavioral and Brain Sciences, 37(2), 141-142.

Frith, C. D., Blakemore, S. J., \& Wolpert, D. M. (2000). Abnormalities in the awareness and control of action. Philosophical Transactions of the Royal Society of London, Series B: Biological Sciences, 355(1404), 1771-1788.

Gaudiano, P., \& Grossberg, S. (1991). Vector associative maps: Unsupervised real-time error-based learning and control of movement trajectories. Neural Networks, 4(2), 147-183.

Haggard, P., \& Chambon, V. (2012). Sense of agency. Current Biology, 22(10), R390-R392.

Hauf, P., Elsner, B., \& Aschersleben, G. (2004). The role of action effects in infants' action control. Psychological Research, 68(2/3), 115125.

Herwig, A., Prinz, W., \& Waszak, F. (2007). Two modes of sensorimotor integration in intention-based and stimulus-based actions. The Quarterly Journal of Experimental Psychology, 60(11), 1540-1554.

Herwig, A., \& Schneider, W. X. (2014). Predicting object features across saccades: Evidence from object recognition and visual search. Journal of Experimental Psychology: General, 143(5), 1903. 
Herwig, A., Weiß, K., \& Schneider, W. X. (2015). When circles become triangular: How transsaccadic predictions shape the perception of shape. Annals of the New York Academy of Sciences, 1339(1), 97105.

Hickey, C., Chelazzi, L., \& Theeuwes, J. (2010). Reward changes salience in human vision via the anterior cingulate. Journal of Neuroscience, 30(33), 11096-11103.

Hickey, C., Chelazzi, L., \& Theeuwes, J. (2014). Reward-priming of location in visual search. PLOS ONE, 9(7), e103372.

Higgins, E. T. (2012). Beyond pleasure and pain: How motivation works. New York, NY: Oxford University Press

Hills, T. T., Todd, P. M., Lazer, D., Redish, A. D., Couzin, I. D., \& Cognitive Search Research Group. (2015). Exploration versus exploitation in space, mind, and society. Trends in Cognitive Sciences, 19(1), 46-54.

Kleiner, M., Brainard, D. H., Pelli, D., Ingling, A., Murray, R., \& Broussard, C. (2007). What's new in Psychtoolbox-3. Perception, 36, 1-16. https://doi.org/10.1068/v070821.

Lavie, N., \& De Fockert, J. (2005). The role of working memory in attentional capture. Psychonomic Bulletin \& Review, 12(4), 669674.

Leotti, L. A., Iyengar, S. S., \& Ochsner, K. N. (2010). Born to choose: The origins and value of the need for control. Trends in Cognitive Sciences, 14, 457-463.

Miranda, A. T., \& Palmer, E. M. (2014). Intrinsic motivation and attentional capture from gamelike features in a visual search task. Behavior Research Methods, 46(1), 159-172.

O'Regan, J. K., \& Noë, A. (2001). A sensorimotor account of vision and visual consciousness. Behavioral and Brain Sciences, 24(5), 939973

Obhi, S. S., \& Haggard, P. (2004). Internally generated and externally triggered actions are physically distinct and independently controlled. Experimental Brain Research, 156(4), 518-523.

Pfister, R., Heinemann, A., Kiesel, A., Thomaschke, R., \& Janczyk, M. (2012). Do endogenous and exogenous action control compete for perception?. Journal of Experimental Psychology: Human Perception and Performance, 38(2), 279.

Pfister, R., \& Janczyk, M. (2013). Confidence intervals for two sample means: Calculation, interpretation, and a few simple rules. Advances in Cognitive Psychology, 9(2), 74.

Philipona, D., O'Regan, J. K., \& Nadal, J. P. (2003). Is there something out there? Inferring space from sensorimotor dependencies. Neural Computation, 15(9), 2029-2049.

Raymond, J. E., \& O'Brien, J. L. (2009). Selective visual attention and motivation: The consequences of value learning in an attentional blink task. Psychological Science, 20(8), 981-988.

Rolf, M., Steil, J. J., \& Gienger, M. (2011). Online goal babbling for rapid bootstrapping of inverse models in high dimensions. In 2011 IEEE International Conference on Development and Learning (ICDL) (Vol. 2, pp. 1-8). New York, NY: IEEE.

Tatler, B. W., Brockmole, J. R., \& Carpenter, R. H. (2017). LATEST: A model of saccadic decisions in space and time. Psychological Review, 124(3), 267.

Theeuwes, J. (1992). Perceptual selectivity for color and form. Perception \& Psychophysics, 51(6), 599-606.

Theeuwes, J. (1994). Stimulus-driven capture and attentional set: selective search for color and visual abrupt onsets. Journal of Experimental Psychology: Human perception and performance, 20(4), 799 .

Wen, W., \& Haggard, P. (2018). Control changes the way we look at the world. Journal of Cognitive Neuroscience, 30(4), 603-619.

White, R. W. (1959). Motivation reconsidered: The concept of competence. Psychological Review, 66(5), 297.

Wolpert, D. M., \& Flanagan, J. R. (2001). Motor prediction. Current Biology, 11(18), R729-R732.

Wolpert, D. M., \& Ghahramani, Z. (2000). Computational principles of movement neuroscience. Nature Neuroscience, 3(11s), 1212.

Publisher's note Springer Nature remains neutral with regard to jurisdictional claims in published maps and institutional affiliations. 